Mathematical Research Letters 3, 123-132 (1996)

\title{
A NOTE ON THE TOTAL CURVATURE OF A KÄHLER MANIFOLD
}

\author{
Wan-Xiong Shi and S.-T. YaU
}

Given a complete manifold with non-negative Ricci curvature, it is a very interesting geometric problem of how curvature decays at infinity. While it is not true that the curvature decays in a strong sense, it is possible that the average of the scalar curvature decays at least linearly. Such a statement is certainly consistent with the Cohn-Vossen inequality which holds for surfaces. The significance of such an inequality is also clear because of its relevance with the work of the first author [1] on the attempt to prove the conjecture of the second author that a complete noncompact Kähler manifold with positive bisectional curvature is biholomorphic to the complex euclidean space.

The purpose of this note is to prove a weaker version of the conjecture for Kähler manifolds.

Theorem 1. Suppose $M$ is a complex $n$-dimensional $(n \geq 3)$ complete noncompact Kähler manifold with bounded and nonnegative holomorphic bisectional curvature such that

$$
R_{\alpha \bar{\alpha} \beta \bar{\beta}} \geq \epsilon R \quad \text { on } M,
$$

where $0<\epsilon<+\infty$ is a constant and $R$ is the scalar curvature. Then we have

$$
\int_{B\left(x_{0}, \gamma\right)} R(x) d x \leq \frac{C(n, \epsilon)}{\gamma^{2}} \operatorname{Vol} B\left(x_{0}, \gamma\right)
$$

for any $x_{0} \in M$ and $0<\gamma<+\infty$, where $B\left(x_{0}, \gamma\right)$ is the geodesic ball of radius $\gamma$ centered at $x_{0}$ and the constant $C(n, \epsilon)$ depends only on $n$ and $\epsilon$.

Since both assumptions and conclusion are scaling invariant, we only need to prove (2) for $\gamma=1$. That is, we only need to prove

Received December 5, 1995.

The first author is supported in part by NSF grant DMS 9403405 . 
Theorem 2. Suppose $M$ is a complex $n$-dimensional $(n \geq 3)$ complete noncompact Kähler manifold such that

$$
\epsilon R \leq R_{\alpha \bar{\alpha} \beta \bar{\beta}} \leq K_{0} \quad \text { on } M,
$$

where $0<\epsilon, K_{0}<+\infty$ are constants. Then

$$
\int_{B\left(x_{0}, 1\right)} R(x) d x \leq c(n, \epsilon) \operatorname{Vol} B\left(x_{0}, 1\right)
$$

for any $x_{0} \in M$, where $c(n, \epsilon)$ depends only on $n$ and $\epsilon$, and is independent of $K_{0}$.

Fix a point $x_{0} \in M$. Suppose $\varphi(x, t)$ is the solution of the heat equation

$$
\begin{cases}\frac{\partial \varphi}{\partial t}=\Delta \varphi & \text { on } M \times[0, \infty) \\ \varphi(x, 0)=\left[\frac{1}{1+\gamma\left(x, x_{0}\right)}\right]^{2 n} & x \in M,\end{cases}
$$

where $\gamma\left(x, x_{0}\right)$ denotes the distance between $x$ and $x_{0}$. We have $\varphi(x, t) \in$ $C^{\infty}(M \times(0, \infty))$,

$$
\begin{cases}\frac{C_{1}}{\left[1+\gamma\left(x, x_{0}\right)\right]^{2 n}} \leq \varphi(x, t) \leq \frac{C_{2}}{\left[1+\gamma\left(x, x_{0}\right)\right]^{2 n}} & \text { on } M \times[0,1] \\ \left|\nabla_{i} \varphi(x, t)\right| \leq \frac{C_{3}}{\left[1+\gamma\left(x, x_{0}\right)\right]^{2 n+1}} & \text { on } M \times[0,1],\end{cases}
$$

where $0<C_{1}, C_{2}, C_{3}<+\infty$ depend only on $n$.

\section{Lemma 3.}

$$
\left|\nabla_{\alpha} \nabla_{\bar{\beta}} \varphi(x, t)\right| \leq \frac{C_{4}}{\left[1+\gamma\left(x, x_{0}\right)\right]^{2 n+1}}\left(\frac{1}{t}\right) \quad \text { on } M \times[0,1],
$$

where $0<C_{4}<+\infty$ depends only on $n$.

Proof. From (5) we have

$$
\begin{aligned}
\frac{\partial}{\partial t}\left|\nabla_{\alpha} \nabla_{\bar{\beta}} \varphi\right|^{2}= & \Delta\left|\nabla_{\alpha} \nabla_{\bar{\beta}} \varphi\right|^{2}-\left|\nabla_{\gamma} \nabla_{\alpha} \nabla_{\bar{\beta}} \varphi\right|^{2}-\left|\nabla_{\bar{\gamma}} \nabla_{\alpha} \nabla_{\bar{\beta}} \varphi\right|^{2} \\
& +2 R_{\alpha \bar{\beta} \gamma \bar{\delta}} \nabla_{\bar{\alpha}} \nabla_{\beta} \varphi \cdot \nabla_{\delta} \nabla_{\bar{\gamma}} \varphi-2 R_{\alpha \bar{\beta}} \nabla_{\beta} \nabla_{\bar{\gamma}} \varphi \cdot \nabla_{\gamma} \nabla_{\bar{\alpha}} \varphi
\end{aligned}
$$

Choose a coordinate system such that at one point

$$
\nabla_{\alpha} \nabla_{\bar{\beta}} \varphi= \begin{cases}0 & \alpha \neq \beta \\ \ell \ell_{\alpha} & \alpha=\beta\end{cases}
$$


Then

$$
\begin{aligned}
& 2 R_{\alpha \bar{\beta} \gamma \bar{\delta}} \nabla_{\bar{\alpha}} \nabla_{\beta} \varphi \cdot \nabla_{\delta} \nabla_{\bar{\gamma}} \varphi-2 R_{\alpha \bar{\beta}} \nabla_{\beta} \nabla_{\bar{\gamma}} \varphi \cdot \nabla_{\gamma} \nabla_{\bar{\alpha}} \varphi \\
& =2 R_{\alpha \bar{\alpha} \gamma \bar{\gamma}} \ell \ell_{\alpha} \ell \ell_{\gamma}-2 R_{\alpha \bar{\alpha}} \ell \ell_{\alpha}^{2} \\
& =-\sum_{\alpha, \beta} R_{\alpha \bar{\alpha} \beta \bar{\beta}} \ell \ell_{\alpha} \ell \ell_{\gamma}-2 R_{\alpha \bar{\alpha}} \ell \ell_{\alpha}^{2} \\
& =-\sum_{\alpha, \beta} R_{\alpha \bar{\alpha} \beta \bar{\beta}}\left(\ell \ell_{\alpha}-\ell \ell_{\beta}\right)^{2} \leq 0 .
\end{aligned}
$$

Thus

$$
\frac{\partial}{\partial t}\left|\nabla_{\alpha} \nabla_{\bar{\beta}} \varphi\right|^{2} \leq \Delta\left|\nabla_{\alpha} \nabla_{\bar{\beta}} \varphi\right|^{2}-\left|\nabla_{\gamma} \nabla_{\alpha} \nabla_{\bar{\beta}} \varphi\right|^{2}-\left|\nabla_{\bar{\gamma}} \nabla_{\alpha} \nabla_{\bar{\beta}} \varphi\right|^{2}
$$

Combining (6) and (10) we can establish (7). For the details, one can see Shi-Yau [2].

Remark. If we do not assume the nonnegativity of the holomorphic bisectional curvature, estimate (7) is still true with the constant $C_{4}$ depends not only on $n$ but also on $K_{0}$.

Let

$$
\psi(x)=\varphi(x, 1) \quad x \in M .
$$

Then

$$
\left|\nabla_{\alpha} \nabla_{\bar{\beta}} \psi(x)\right| \leq \frac{C_{4}}{(1+\gamma)^{2 n+1}} .
$$

For the remaining part of this note, we always denote

$$
\gamma=\gamma(x)=\gamma\left(x, x_{0}\right), \quad x \in M .
$$


Suppose $U(x)$ is the function defined by

$$
U(x)=-\int_{M} G(x, y) \psi(y) d y, \quad x \in M
$$

where $G(x, y)>0$ is the Green function on $M$. Then

$$
\Delta U(x)=\psi(x) \quad x \in M
$$

If $G(x, y)$ does not exist on $M$, we can use elliptic equation theory to solve (18). Thus (13), (14), and (15) give

$$
\left\{\begin{array}{l}
\frac{C_{1}}{(1+\gamma)^{2 n}} \leq \Delta U \leq \frac{C_{2}}{(1+\gamma)^{2 n}} \\
\left|\nabla_{\alpha}(\Delta U)\right| \leq \frac{C_{3}}{(1+\gamma)^{2 n+1}} \\
\left|\nabla_{\alpha} \nabla_{\bar{\beta}}(\Delta U)\right| \leq \frac{C_{4}}{(1+\gamma)^{2 n+1}} .
\end{array} \quad \text { on } M\right.
$$

Since $R_{i j} \geq 0$ on $M$, we have

$$
\frac{C(n) \gamma(x, y)^{2}}{\operatorname{Vol} B(x, \gamma(x, y))} \leq G(x, y) \leq \frac{\widetilde{C(n)} \gamma(x, y)^{2}}{\operatorname{Vol} B(x, \gamma(x, y))}, \quad \forall x, y \in M
$$

where $0<C(n), \widetilde{C(n)}<+\infty$ depend only on $n$.

Combining (13), (17), (18), (19) and (20) we can show that

$$
-\frac{C_{7}(\gamma+1)^{2} \operatorname{Vol} B\left(x_{0}, 1\right)}{\operatorname{Vol} B\left(x_{0}, \gamma+1\right)} \leq U(x) \leq-\frac{C_{8}(\gamma+1)^{2} \operatorname{Vol} B\left(x_{0}, 1\right)}{\operatorname{Vol} B\left(x_{0}, \gamma+1\right)} \quad \forall x \in M
$$

$$
\begin{gathered}
\left|\nabla_{\alpha} U(x)\right| \leq \frac{C_{9}(\gamma+1)^{2} \operatorname{Vol} B\left(x_{0}, 1\right)}{\operatorname{Vol} B\left(x_{0}, \gamma+1\right)} \quad x \in M \\
\left|\nabla_{\alpha} \nabla_{\bar{\beta}} U(x)\right| \leq \frac{C_{9}(\gamma+1)^{2} \operatorname{Vol} B\left(x_{0}, 1\right)}{\operatorname{Vol} B\left(x_{0}, \gamma+1\right)} \quad x \in M,
\end{gathered}
$$

where $0<C_{7}, C_{8}, C_{9}<+\infty$ depend only on $n$. The proof of (23) is similar to the proof of (7). The only difference is to replace $\frac{\partial \varphi}{\partial t}$ in $(5)$ by $\psi(x)$ in (18). 
Using the interchange formula for covariant derivatives, we have (convention: $\left.\Delta=\frac{1}{2} \nabla_{\alpha} \nabla_{\bar{\alpha}}+\frac{1}{2} \nabla_{\bar{\alpha}} \nabla_{\alpha}\right)$

$$
\begin{gathered}
\Delta\left(\nabla_{\bar{\beta}} \nabla_{\gamma} U\right)=\nabla_{\bar{\beta}} \nabla_{\gamma}(\Delta U)+\frac{1}{2} R_{\theta \bar{\beta}} \nabla_{\bar{\theta}} \nabla_{\gamma} U \\
\quad+\frac{1}{2} R_{\gamma \bar{\theta}} \nabla_{\bar{\beta}} \nabla_{\theta} U-R_{\alpha \bar{\beta} \gamma \bar{\theta}} \nabla_{\bar{\alpha}} \nabla_{\theta} U \\
\Delta\left|\nabla_{\bar{\beta}} \nabla_{\gamma} U\right|= \\
2 \operatorname{Re}\left\{\nabla_{\beta} \nabla_{\bar{\gamma}} U \cdot \nabla_{\bar{\beta}} \nabla_{\gamma}(\Delta U)\right. \\
+\left|\nabla_{\alpha} \nabla_{\bar{\beta}} \nabla_{\gamma} U\right|^{2}+\left|\nabla_{\bar{\alpha}} \nabla_{\bar{\beta}} \nabla_{\gamma} U\right|^{2} \\
\left.+2 R_{\theta \bar{\beta}} \nabla_{\bar{\theta}} \nabla_{\gamma} U \cdot \nabla_{\beta} \nabla_{\bar{\gamma}} U-2 R_{\alpha \bar{\beta} \gamma \bar{\theta}} \nabla_{\bar{\alpha}} \nabla_{\theta} U \cdot \nabla_{\beta} \nabla_{\bar{\gamma}} U\right\} .
\end{gathered}
$$

Thus

$$
\begin{aligned}
& 2 \int_{M} \operatorname{Re}\left\{\nabla_{\beta} \nabla_{\bar{\gamma}} U \cdot \nabla_{\bar{\beta}} \nabla_{\gamma}(\Delta U)\right\} d x \\
& +\int_{M}\left[\left|\nabla_{\alpha} \nabla_{\bar{\beta}} \nabla_{\gamma} U\right|^{2}+\left|\nabla_{\bar{\alpha}} \nabla_{\bar{\beta}} \nabla_{\gamma} U\right|^{2}\right] d x \\
& +2 \int_{M}\left[R_{\theta \bar{\beta}} \nabla_{\bar{\theta}} \nabla_{\gamma} U \cdot \nabla_{\beta} \nabla_{\bar{\gamma}} U-R_{\alpha \bar{\beta} \gamma \bar{\theta}} \nabla_{\bar{\alpha}} \nabla_{\theta} U \cdot \nabla_{\beta} \nabla_{\bar{\gamma}} U\right] d x \\
& =0 .
\end{aligned}
$$

Choose a coordinate system such that at one point

$$
\nabla_{\alpha} \nabla_{\bar{\beta}} U= \begin{cases}0 & \alpha \neq \beta \\ \lambda_{\alpha} & \alpha=\beta\end{cases}
$$

Then

$$
\begin{aligned}
& R_{\theta \bar{\beta}} \nabla_{\bar{\theta}} \nabla_{\gamma} U \cdot \nabla_{\beta} \nabla_{\bar{\gamma}} U-R_{\alpha \bar{\beta} \gamma \bar{\theta}} \nabla_{\bar{\alpha}} \nabla_{\theta} U \cdot \nabla_{\beta} \nabla_{\bar{\gamma}} U \\
& =\sum_{\alpha, \beta} R_{\alpha \bar{\alpha} \beta \bar{\beta}}\left(\lambda_{\alpha}-\lambda_{\beta}\right)^{2} \geq 0 .
\end{aligned}
$$

(24) can be written as

$$
\begin{aligned}
& 2 \int_{M} \operatorname{Re}\left\{\nabla_{\beta} \nabla_{\bar{\gamma}} U \cdot \nabla_{\bar{\beta}} \nabla_{\gamma}(\Delta U)\right\} d x \\
& +\int_{M}\left[\left|\nabla_{\alpha} \nabla_{\bar{\beta}} \nabla_{\gamma} U\right|^{2}+\left|\nabla_{\bar{\alpha}} \nabla_{\bar{\beta}} \nabla_{\gamma} U\right|^{2}\right] d x \\
& +2 \int_{M} \sum_{\alpha, \beta} R_{\alpha \bar{\alpha} \beta \bar{\beta}}\left(\lambda_{\alpha}-\lambda_{\beta}\right)^{2} d x=0 .
\end{aligned}
$$


But from (19) and (23) we have

$$
\begin{aligned}
& 2\left|\int_{M} \operatorname{Re}\left\{\nabla_{\beta} \nabla_{\bar{\gamma}} U \cdot \nabla_{\bar{\beta}} \nabla_{\gamma}(\Delta U)\right\} d x\right| \\
& \leq 2 \int_{M}\left|\nabla_{\beta} \nabla_{\bar{\gamma}} U\right| \cdot\left|\nabla_{\bar{\beta}} \nabla_{\gamma}(\Delta U)\right| d x \\
& \leq \int_{M} \frac{2 C_{4} C_{9} \operatorname{Vol} B\left(x_{0}, 1\right)}{(1+\gamma)^{2 n+1} \operatorname{Vol} B\left(x_{0}, \gamma+1\right)} d x \leq C_{10} \operatorname{Vol} B\left(x_{0}, 1\right),
\end{aligned}
$$

where $0<C_{10}<+\infty$ depends only on $n$.

Combining (27) and (28) we get

$$
\int_{M}\left\{\left|\nabla_{\alpha} \nabla_{\bar{\beta}} \nabla_{\gamma} U\right|^{2}+\left|\nabla_{\bar{\alpha}} \nabla_{\bar{\beta}} \nabla_{\gamma} U\right|^{2}\right\} d x \leq C_{10} \operatorname{Vol} B\left(x_{0}, 1\right)
$$

$$
2 \int_{M} \sum_{\alpha, \beta} R_{\alpha \bar{\alpha} \beta \bar{\beta}}\left(\lambda_{\alpha}-\lambda_{\beta}\right)^{2} d x \leq C_{10} \operatorname{Vol} B\left(x_{0}, 1\right) .
$$

On the other hand, we have

$$
\begin{aligned}
& \int_{M} R(\Delta U)^{2} d x=\int_{M} R \Delta U \cdot \Delta U d x \\
& =\int_{M} R \Delta U \cdot \nabla_{\alpha} \nabla_{\bar{\alpha}} U d x \\
& =-\int_{M} R \nabla_{\alpha}(\Delta U) \cdot \nabla_{\bar{\alpha}} U d x-\int_{M} \nabla_{\alpha} R \cdot \Delta U \cdot \nabla_{\bar{\alpha}} U d x \\
& =-\int_{M} R \nabla_{\alpha} \nabla_{\beta} \nabla_{\bar{\beta}} U \cdot \nabla_{\bar{\alpha}} U d x-\int_{M} \nabla_{\gamma} R_{\alpha \bar{\gamma}} \cdot \Delta U \cdot \nabla_{\bar{\alpha}} U d x \\
& =-\int_{M} R \nabla_{\beta} \nabla_{\alpha} \nabla_{\bar{\beta}} U \cdot \nabla_{\bar{\alpha}} U d x+\int_{M} R_{\alpha \bar{\gamma}} \nabla_{\gamma}\left[\Delta U \cdot \nabla_{\bar{\alpha}} U\right] d x \\
& =\int_{M} \nabla_{\alpha} \nabla_{\bar{\beta}} U \cdot \nabla_{\beta}\left[R \nabla_{\bar{\alpha}} U\right] d x+\int_{M} R_{\alpha \bar{\gamma}} \nabla_{\gamma}\left[\Delta U \cdot \nabla_{\bar{\alpha}} U\right] d x \\
& =\int_{M} R \nabla_{\alpha} \nabla_{\bar{\beta}} U \cdot \nabla_{\beta} \nabla_{\bar{\alpha}} U d x+\int_{M} \nabla_{\beta} R \cdot \nabla_{\alpha} \nabla_{\bar{\beta}} U \cdot \nabla_{\bar{\alpha}} U d x \\
& +\int_{M} R_{\alpha \bar{\gamma}} \nabla_{\gamma} \nabla_{\bar{\alpha}} U \cdot \Delta U d x+\int_{M} R_{\alpha \bar{\gamma}} \nabla_{\gamma}(\Delta U) \cdot \nabla_{\bar{\alpha}} U d x .
\end{aligned}
$$




$$
\begin{aligned}
& \int_{M} \nabla_{\beta} R \cdot \nabla_{\alpha} \nabla_{\bar{\beta}} U \cdot \nabla_{\bar{\alpha}} U d x \\
& =\int_{M} \nabla_{\gamma} R_{\beta \bar{\gamma}} \cdot \nabla_{\alpha} \nabla_{\bar{\beta}} U \cdot \nabla_{\bar{\alpha}} U d x \\
& =-\int_{M} R_{\beta \bar{\gamma}} \nabla_{\gamma} \nabla_{\alpha} \nabla_{\bar{\beta}} U \cdot \nabla_{\underline{\alpha}} U d x-\int_{M} R_{\beta \bar{\gamma}} \nabla_{\alpha} \nabla_{\bar{\beta}} U \cdot \nabla_{\gamma} \nabla_{\bar{\alpha}} U d x \\
& =-\int_{M} R_{\beta \bar{\gamma}} \nabla_{\alpha} \nabla_{\gamma} \nabla_{\bar{\beta}} U \cdot \nabla_{\bar{\alpha}} U d x-\int_{M} R_{\beta \bar{\gamma}} \nabla_{\alpha} \nabla_{\bar{\beta}} U \cdot \nabla_{\gamma} \nabla_{\bar{\alpha}} U d x \\
& =\int_{M} \nabla_{\gamma} \nabla_{\bar{\beta}} U \cdot \nabla_{\alpha}\left[R_{\beta \bar{\gamma}} \nabla_{\bar{\alpha}} U\right] d x-\int_{M} R_{\beta \bar{\gamma}} \nabla_{\alpha} \nabla_{\bar{\beta}} U \cdot \nabla_{\gamma} \nabla_{\bar{\alpha}} U d x \\
& =\int_{M} \nabla_{\alpha} R_{\beta \bar{\gamma}} \cdot \nabla_{\gamma} \nabla_{\bar{\beta}} U \cdot \nabla_{\bar{\alpha}} U d x+\int_{M} R_{\beta \bar{\gamma}} \nabla_{\gamma} \nabla_{\bar{\beta}} U \cdot \Delta U d x \\
& -\int_{M} R_{\beta \bar{\gamma}} \nabla_{\alpha} \nabla_{\bar{\beta}} U \cdot \nabla_{\gamma} \nabla_{\bar{\alpha}} U d x \\
& =\int_{M} \nabla_{\beta} R_{\alpha \bar{\gamma}} \cdot \nabla_{\gamma} \nabla_{\bar{\beta}} U \cdot \nabla_{\bar{\alpha}} U d x+\int_{M} R_{\beta \bar{\gamma}} \nabla_{\gamma} \nabla_{\bar{\beta}} U \cdot \Delta U d x \\
& -\int_{M} R_{\beta \bar{\gamma}} \nabla_{\alpha} \nabla_{\bar{\beta}} U \cdot \nabla_{\gamma} \nabla_{\bar{\alpha}} U d x \\
& =-\int_{M} R_{\alpha \bar{\gamma}} \nabla_{\gamma}(\Delta U) \cdot \nabla_{\bar{\alpha}} U d x-\int_{M} R_{\alpha \bar{\gamma}} \nabla_{\gamma} \nabla_{\bar{\beta}} U \cdot \nabla_{\beta} \nabla_{\bar{\alpha}} U d x \\
& +\int_{M} R_{\beta \bar{\gamma}} \nabla_{\gamma} \nabla_{\bar{\beta}} U \cdot \Delta U d x-\int_{M} R_{\beta \bar{\gamma}} \nabla_{\alpha} \nabla_{\bar{\beta}} U \cdot \nabla_{\gamma} \nabla_{\bar{\alpha}} U d x .
\end{aligned}
$$

Combining (31) and (32) we get

$$
\begin{aligned}
\int_{M} R(\Delta U)^{2} d x= & \int_{M} R \nabla_{\alpha} \nabla_{\bar{\beta}} U \cdot \nabla_{\bar{\alpha}} \nabla_{\beta} U d x \\
& +2 \int_{M} R_{\alpha \bar{\gamma}} \nabla_{\gamma} \nabla_{\bar{\alpha}} U \cdot \Delta U d x \\
& -2 \int_{M} R_{\alpha \bar{\gamma}} \nabla_{\gamma} \nabla_{\bar{\beta}} U \cdot \nabla_{\beta} \nabla_{\bar{\alpha}} U d x .
\end{aligned}
$$

If we define a function

$$
\begin{aligned}
F(x)= & R(\Delta U)^{2}-R \nabla_{\alpha} \nabla_{\bar{\beta}} U \cdot \nabla_{\bar{\alpha}} \nabla_{\beta} U \\
& +2 R_{\alpha \bar{\gamma}} \nabla_{\gamma} \nabla_{\bar{\beta}} U \cdot \nabla_{\beta} \nabla_{\bar{\alpha}} U \\
& -2 R_{\alpha \bar{\gamma}} \nabla_{\gamma} \nabla_{\bar{\alpha}} U \cdot \Delta U .
\end{aligned}
$$

Then

$$
\int_{M} F(x) d x=0
$$


With the coordinate (25) we know that

$$
\left|\nabla_{\alpha} \nabla_{\bar{\beta}} U\right|=\sqrt{\sum_{\alpha} \lambda_{\alpha}^{2}} .
$$

Define $\Omega \subseteq M$ such that

$$
\Omega=\left\{x \in M|| \lambda_{\alpha}-\frac{\left|\nabla_{\beta} \nabla_{\bar{\gamma}} U\right|}{\sqrt{n}} \mid \leq \frac{\left|\nabla_{\beta} \nabla_{\bar{\gamma}} U\right|}{(2 n+10)^{5}} \quad \text { for } \alpha=1,2, \cdots, n\right\} .
$$

It is easy to see that for any $x \in M \backslash \Omega$, there exist $\alpha$ and $\beta$ (say $\alpha=1, \beta=$ 2) such that

$$
\left|\lambda_{1}-\lambda_{2}\right| \geq \frac{\left|\nabla_{\beta} \nabla_{\bar{\gamma}} U\right|}{(2 n+10)^{5}}
$$

Thus

(38)

$$
\begin{aligned}
\sum_{\alpha, \beta} R_{\alpha \bar{\alpha} \beta \bar{\beta}}\left(\lambda_{\alpha}-\lambda_{\beta}\right)^{2} \geq & R_{1 \overline{1} 2 \overline{2}} \frac{\left|\nabla_{\alpha} \nabla_{\bar{\beta}} U\right|^{2}}{(2 n+10)^{10}} \geq \frac{\epsilon}{(2 n+10)^{10}} R\left|\nabla_{\alpha} \nabla_{\bar{\beta}} U\right|^{2} \\
\forall x \in M \backslash \Omega & \\
\int_{M \backslash \Omega} R\left|\nabla_{\alpha} \nabla_{\bar{\beta}} U\right|^{2} d x & \leq \frac{(2 n+10)^{10}}{\epsilon} \int_{M \backslash \Omega} \sum_{\alpha, \beta} R_{\alpha \bar{\alpha} \beta \bar{\beta}}\left(\lambda_{\alpha}-\lambda_{\beta}\right)^{2} d x \\
\leq & \frac{(2 n+10)^{10}}{\epsilon} \int_{M} \sum_{\alpha, \beta} R_{\alpha \bar{\alpha} \beta \bar{\beta}}\left(\lambda_{\alpha}-\lambda_{\beta}\right)^{2} d x .
\end{aligned}
$$

Combining (30) and (39) we know that

$$
\int_{M \backslash \Omega} R\left|\nabla_{\alpha} \nabla_{\bar{\beta}} U\right|^{2} d x \leq \frac{(2 n+10)^{10}}{2 \epsilon} C_{10} \operatorname{Vol} B\left(x_{0}, 1\right) .
$$

It is easy to see that

$$
|F(x)| \leq C_{11}(n) R\left|\nabla_{\alpha} \nabla_{\bar{\beta}} U\right|^{2} \quad \forall x \in M .
$$

Thus

$$
\begin{aligned}
& \left|\int_{M \backslash \Omega} F(x) d x\right| \leq \int_{M \backslash \Omega}|F(x)| d x \leq \\
& \leq C_{11} \int_{M \backslash \Omega} R\left|\nabla_{\alpha} \nabla_{\bar{\beta}} U\right|^{2} d x \leq C_{12}(n, \epsilon) \operatorname{Vol} B\left(x_{0}, 1\right) .
\end{aligned}
$$


From (35) we get

$$
\int_{\Omega} F(x) d x=-\int_{M \backslash \Omega} F(x) d x
$$

Thus from (42)

$$
\left|\int_{\Omega} F(x) d x\right| \leq C_{12}(n, \epsilon) \operatorname{Vol} B\left(x_{0}, 1\right) .
$$

Since on $\Omega$ we have

$$
\lambda_{\alpha} \sim \frac{\left|\nabla_{\beta} \nabla_{\bar{\gamma}} U\right|}{\sqrt{n}} \quad \alpha=1,2, \cdots, n .
$$

From (34) we have

$$
\begin{aligned}
F(x) & \sim R \cdot n\left|\nabla_{\beta} \nabla_{\bar{\gamma}} U\right|^{2}-R\left|\nabla_{\beta} \nabla_{\bar{\gamma}} U\right|^{2}+ \\
& +2 \sum_{\alpha} R_{\alpha \bar{\alpha}} \cdot \frac{1}{n}\left|\nabla_{\beta} \nabla_{\bar{\gamma}} U\right|^{2}-2 \sum_{\alpha} R_{\alpha \bar{\alpha}}\left|\nabla_{\beta} \nabla_{\bar{\gamma}} U\right|^{2}, \\
F(x) & \sim \sum_{\alpha} R_{\alpha \bar{\alpha}}\left(n-3+\frac{2}{n}\right)\left|\nabla_{\beta} \nabla_{\bar{\gamma}} U\right|^{2}, \quad \forall x \in \Omega .
\end{aligned}
$$

Since $n \geq 3$, we have $n-3+\frac{2}{n} \geq \frac{2}{n}$. (44) is not precise. Precisely we have

$$
F(x) \geq \frac{1}{n} R\left|\nabla_{\beta} \nabla_{\bar{\gamma}} U\right|^{2} \quad x \in \Omega .
$$

(43), (45) $\Longrightarrow$

$$
\int_{\Omega} R\left|\nabla_{\beta} \nabla_{\bar{\gamma}} U\right|^{2} d x \leq C_{13}(n, \epsilon) \operatorname{Vol} B\left(x_{0}, 1\right) .
$$

(40), (46) $\Longrightarrow$

$$
\int_{M} R\left|\nabla_{\beta} \nabla_{\bar{\gamma}} U\right|^{2} d x \leq C_{14}(n, \epsilon) \operatorname{Vol} B\left(x_{0}, 1\right) .
$$

$(19) \Longrightarrow$

$$
\left|\nabla_{\beta} \nabla_{\bar{\gamma}} U\right|^{2} \geq \frac{C_{1}^{2}}{n(1+\gamma)^{4 n}}, \quad x \in M
$$

$(47),(48) \Longrightarrow$

$$
\int_{M} \frac{R(x) d x}{\left[1+\gamma\left(x, x_{0}\right)\right]^{4 n}} \leq C_{15}(n, \epsilon) \operatorname{Vol} B\left(x_{0}, 1\right) .
$$

Thus finally we have

$$
\int_{B\left(x_{0}, 1\right)} R(x) d x \leq C_{16}(n, \epsilon) \operatorname{Vol} B\left(x_{0}, 1\right) .
$$

Theorem 2 is proved. 


\section{References}

1. W. X. Shi, Ricci deformation of the metric on complete noncompact Kähler manifolds, Ph.D. Thesis; Harvard University (1990).

2. W.X. Shi and S.-T. Yau, Harmonic maps on complete noncompact Riemannian manifolds, A tribute to Ilya Bakelman, ed. by I.R. Bakelman, S. A. Fulling and S. D. Taliaferro, vol. 3, Discourses in mathematics and its applications, Texas A\&M University, College Station, TX, (1994), pp. 79-120.

Department of Mathematics, Harvard University, Cambridge M A 02138

E-mail address: yau@math.harvard.edu

Department of Mathematics, Purdue Univerity, West Lafayette, In 47907

E-mail address: wxshi@math.purdue.edu 